\title{
Survey Mode Effects on Objective and Subjective Questions: Evidence from the Labour Force Survey
}

\author{
Joachim Schork ${ }^{1}$, Cesare A.F. Riillo ${ }^{1}$, and Johann Neumayr ${ }^{1}$
}

\begin{abstract}
Web questionnaires are increasingly used to complement traditional data collection in mixed mode surveys. However, the utilization of web data raises concerns whether web questionnaires lead to mode-specific measurement bias. We argue that the magnitude of measurement bias strongly depends on the content of a variable. Based on the Luxembourgish Labour Force Survey, we investigate differences between web and telephone data in terms of objective (i.e., Employment Status) and subjective (i.e., Wage Adequacy and Job Satisfaction) variables. To assess whether differences in outcome variables are caused by sample composition or mode-specific measurement bias, we apply a coarsened exact matching that approximates randomized experiments by reducing dissimilarities between web and telephone samples. We select matching variables with a combination of automatic variable selection via random forest and a literature-driven selection. The results show that objective variables are not affected by mode-specific measurement bias, but web participants report lower satisfaction-levels on subjective variables than telephone participants. Extensive supplementary analyses confirm our results. The present study supports the view that the impact of survey mode depends on the content of a survey and its variables.
\end{abstract}

Key words: Web survey; telephone survey; mode effects; coarsened exact matching; measurement bias.

\section{Introduction}

Statistical surveys provide an important basis for evaluation and decision making across many areas. For many decades, survey data was mainly collected by interviewers (e.g., telephone interviews or face-to-face interviews) or via self-administered mail questionnaires. Traditional data collection increasingly faces challenges such as declining response rates, less availability of traditional ways of collecting data (e.g., fixed-line telephone numbers), or the demand of more timely data dissemination, leading to lowered

1 Statec Luxembourg, B.P. 304, L-2013 Luxembourg, 2013 Luxembourg. Emails: joachimschork@ googlemail.com, cesare.riillo@statec.etat.lu and johann.neumayr@statec.etat.lu

Acknowledgments: The authors are grateful to Guillaume Osier for his helpful discussions and comments. We would also like to thank three anonymous referees and editors for their careful and constructive reviews. Furthermore, we appreciate the feedback of the participants at the Labour Force Survey Workshop, 17-18 May 2018 in Reykjavik, Iceland; the Q2018 Conference, 26-29 June 2018 in Kraków, Poland; the Workshop on Household Survey Nonresponse, 22-24 August 2018 in Budapest, Hungary; the 10e Colloque Francophone Sur Les Sondages, 24-26 October 2018 in Lyon, France; the GOR Conference, 6-8 March 2019 in Cologne, Germany; and the Workshop on mixed-mode surveys, 16-17 May 2019 in Luxembourg City, Luxembourg. Cesare A.F. Riillo gratefully acknowledges the support of the Observatoire de la Compétitivité, Ministère de l'Economie, DG Compétitivité, Luxembourg, and STATEC, the National Statistical Institute of Luxembourg. Views and opinions expressed in this article are those of the authors and do not reflect those of STATEC or STATEC Research and funding partners. 
data quality and higher data collection costs (De Leeuw 2018; Dillman 2017; Groves 2005).

At the same time, technological improvements and the rapidly increasing number of households with internet access provide data collectors with web questionnaires as additional data collection mode. Compared to traditional collection modes, internet surveys potentially have numerous advantages (Bianchi et al. 2017; Couper 2011; Fan and Yan 2010; Greene et al. 2008; De Leeuw 2005). Web questionnaires are often more costefficient; faster to process and disseminate; less time-consuming and more convenient for participants; easy to deliver to a large number of participants; and they provide better coverage of many individuals that are difficult to access via traditional channels. For these reasons, web questionnaires are an attractive tool either as exclusive data collection mode or complementary to traditional methods.

The usage of web surveys raises concerns whether collecting data online leads to lower data quality, initially because of low internet prevalence among the population. Nowadays, the increasing number of people accessing the internet mitigates population coverage issues, but concerns remain about lower data quality of online surveys due to other sources such as mode-specific measurement bias, that is, significantly different responses of the same participant when using different survey modes. This study aims to contribute to the growing literature on mixed mode surveys by assessing mode-specific measurement bias using data collected in the Luxembourgish Labour Force Survey (LFS), the largest European survey on employment related variables. The findings of our study have important implications for the comparability of official statistics such as the LFS across countries and times.

Measurement bias can be caused by many different reasons, such as social desirability; a tendency to respond similarly to questions of the same topic; a preference to give positive answers; and a difference in responses depending on the order of questions and answer possibilities (see Dillman and Christian 2005; Sarracino et al. 2017 for detailed overviews). Measurement bias may affect both web and telephone data, but the magnitude of bias might be different in one mode compared to the other.

Measurement bias differing across modes can decrease the quality of inference in sample surveys. Indeed, common calibration estimators that correct for selectivity generally assume that measurement bias is constant across modes (Buelens and Van den Brakel 2015). Our work aims to quantify mode differences and argues that the magnitude of mode-specific measurement bias strongly depends on the content of a variable. More precisely, we examine mode-specific measurement bias in terms of objective (i.e., factual content that is clear to participants and precisely defined) and subjective (i.e., self-assessed personal opinions) variables. We argue that objective variables are less affected by modespecific measurement bias than subjective variables.

The present article is structured as follows. Section 2 discusses previous research. Section 3 describes the data collection and processing. Section 4 illustrates our methodological approach, that is, coarsened exact matching supported by automatic variable selection via random forest and a literature-driven selection. In Section 5, we present the results by comparing sample composition and outcome variables before and after matching. Section 6 shows several supplementary analyses, which evaluate the 
validity and stability of the results of the present study. In Section 7 we conclude with a summary and an outlook for further research.

\section{Background}

Literature generally decomposes mode-specific bias into three groups: coverage, nonresponse, and measurement bias (Felderer et al. 2019; Roberts and Vandenplas 2017; Schouten and Van der Laan 2014; Vannieuwenhuyze et al. 2014). Mode-specific coverage bias results from a mismatch between sampling frame and target population that leads to systematic over- or underrepresentation in the sample. Both web and telephone are affected by coverage bias, for example as result of limited access to a computer with internet or due to the lack of a registered telephone number. Mode-specific nonresponse bias results from differences in contactability and response behavior over survey modes. Even though web surveys usually suffer from relatively high unit nonresponse, they might have higher response rates in specific groups of the target population in comparison to telephone surveys (Fricker et al. 2005; Manfreda et al. 2008; Sinclair et al. 2012). In the present study, we focus on mode-specific measurement bias and isolate measurement bias from coverage and nonresponse bias.

Researchers have investigated mode-specific measurement bias across several domains with varying results using randomized experiments and re-weighting approaches (Körner and Liersch 2014; Pohjanpää 2014; Schouten and Van der Laan 2014). In a meta-analysis, Ye et al. (2011) compared 18 research papers about mixed mode differences and found that telephone participants are more likely to select extremely positive answers than participants collected by other modes. Lugtig et al. (2011) found that web participants reported greater negative effects due to environmental pollution than participants of a telephone sample. Sarracino et al. (2017) measured significant, but only slightly lower well-being for web participants compared to telephone participants. Bell et al. (2011) compared four different survey modes and observed no significant differences in the evaluation of the water quality between web participants and those who were invited to give their responses at a central location. Participants that received a questionnaire on a computer disk and participants that were contacted in a shopping mall, however, reported significantly higher values than web and central location participants.

Research has also examined the impact of mixed collection modes on measuring employment related variables. Within the framework of a European Statistical System Network (ESSnet) project on data collection for social surveys using multiple modes, several National Statistical Institutes (NSIs) investigated mode-specific measurement bias on the basis of different social surveys such as the LFS (Blanke and Luiten 2014; Luiten and Blanke 2015). In a comparison of LFS data collected by web and other modes, employment variables such as working hours, status in employment, and education level revealed significant measurement bias (Körner and Liersch 2014; Schouten and Van der Laan 2014). In contrast, differences in the variable Employment Status could mostly be explained by auxiliary variables such as sex and age, indicating that Employment Status is not affected by mode-specific measurement bias (Luiten and Blanke 2015). In a randomized experiment, Helppie-McFall and Hsu (2017) also observed a high similarity of employment related variables collected by different modes. The authors compared 
responses via web and mail to variables such as household income or understanding of stock markets and found no significant differences in the response distributions of the two groups. Felderer et al. (2019) compared web and telephone data with administrative records and found measurement bias for variables such as regular employment, marginal employment, and mean monthly labour income. For regular employment and marginal employment, however, the differences between web and telephone data where not statistically significant.

The varying findings across mixed mode research suggest that the magnitude of measurement bias strongly depends on the specific topic of a survey and the content of its variables. Dillman and Christian (2005) argue that respondents may not have a readymade answer to questions about opinions, leading to a higher risk that the answering process is affected by the way data is collected. In a similar argument, Tourangeau et al. (2000) differentiate between factual and attitudinal variables and present a psychological model that describes the answering process for the different types of variables. Following this framework, Schonlau et al. (2003) found that responses to attitudinal variables about health care are nine times more likely to be significantly different between web and telephone samples than factual variables.

Hence, mode-specific measurement bias needs to be examined separately for different research domains and variables. In the present study, we examine measurement bias in terms of objective (i.e., factual content that is clear to participants and precisely defined) and subjective (i.e., self-assessed personal opinions) variables. We hypothesize that objective variables are less affected by mode-specific measurement bias than subjective variables. The response to objective variables is typically clearer and more fixed in participants' minds and, hence, we assume that responses to objective variables are less likely to be affected by an interviewer or the self-administered nature of a web questionnaire. In contrast, participants usually have to think more deeply before they are able to respond to subjective variables and this thinking process might be affected by the conditions during the data collection.

We test our hypotheses by applying statistical matching to the Luxembourgish LFS. Randomized experiments are considered as best practice, when mode effects are evaluated, since they disentangle mode-specific measurement bias from other sources of differences between web and telephone data under certain conditions. However, when access to randomized data is limited, statistical matching methods can be used to approximate randomized experiments (Stuart 2010). Matching methods are particularly suitable for observational data when researchers cannot completely control experiments. Even when respondents can be assigned to a particular mode, they cannot be forced to respond to a survey. Simple comparisons between modes in randomized data may therefore suffer from mode-specific nonresponse bias.

We use a coarsened exact matching (CEM) to match web and telephone samples, since CEM generally provides the best trade-off between auxiliary variables' imbalance and sample size of matched participants, when compared to other matching methods such as Mahalanobis or propensity score matching (Iacus et al. 2012). Furthermore, CEM is successfully implemented in many domains, such as innovation (Aggarwal and Hsu 2013) or environmental research (Riillo 2017) and was already applied by Sidney et al. (2015) to 
measure mode-specific measurement bias in well-being variables. Subsection 4.1 describes the application of CEM in more detail.

\section{Data}

The present study is based on data from the Luxembourgish LFS, which is designed to collect employment related variables. The LFS is a quarterly survey of a sample of individuals and is the most comprehensive survey in Luxembourg in terms of sample size. The LFS follows a rotating panel design, in which households remain in the sample for five consecutive quarters. The target population is the non-institutionalized population (i.e., excluding people living in institutional households, such as military bases, youth centers and retirement homes) with an age of at least 15 years. The sample is drawn via simple random sampling from the central population register (Registre national des personnes physiques, RNPP). Consistently dropping response rates led to the introduction of a mixed mode sampling design in 2015, consisting of Computer Assisted Telephone Interviewing (CATI) and Computer Assisted Web Interviewing (CAWI). As suggested in literature, a unified questionnaire design is used (Dillman 2017). The wording and structure of the questions are not altered in the different modes to rule out measurement differences across modes.

Since 2015, the strategy for data collection (in the first wave) is as follows: When the sample is drawn from the population register, it is checked for each sample unit in an online search whether a phone number exists in the official telephone directories (Editus Luxembourg 2018). Sampling units for which a telephone number is available are approached by telephone. Remaining sampling units are approached via an invitation letter containing the internet address for the web questionnaire and a personal login to gain access to the questionnaire. A household in the telephone sample is classified as a nonresponse if interviewers are unable to obtain a response after 30 contact attempts. Web participants who do not respond to the online questionnaire within two weeks receive a reminder letter. If they still do not respond, they are considered as unit nonresponse. The response rate for the combined data of the years 2015-2017 is on average 49\% for the web sample and $61 \%$ for the telephone sample. Participants have the possibility to change from web to telephone or from telephone to web, respectively. However, since such a change requires participants to be proactive, the number of changes is very low $(<1 \%)$. It is therefore important to note that the telephone sub-sample almost exclusively contains participants with a registered telephone number, that is, web and telephone samples are not allocated randomly. In the mixed mode analysis of the present article, we group respondents by their final response mode.

As result of the LFS data collection strategy, the web and telephone sample compositions are different. Figure 1 shows a cross tabulation of the variables age, sex, and nationality. The share of the population (based on the Luxembourgish Census) for each of these groups is presented on the right column of the table on the left. The graphics in the middle and the right illustrate weighted differences to the population of web and telephone samples of the LFS 2017, that is, under-represented groups are displayed by a negative bar and over-represented groups are displayed by a positive bar. The figure reveals mainly two systematic differences of web and telephone samples to the population: First, age groups 

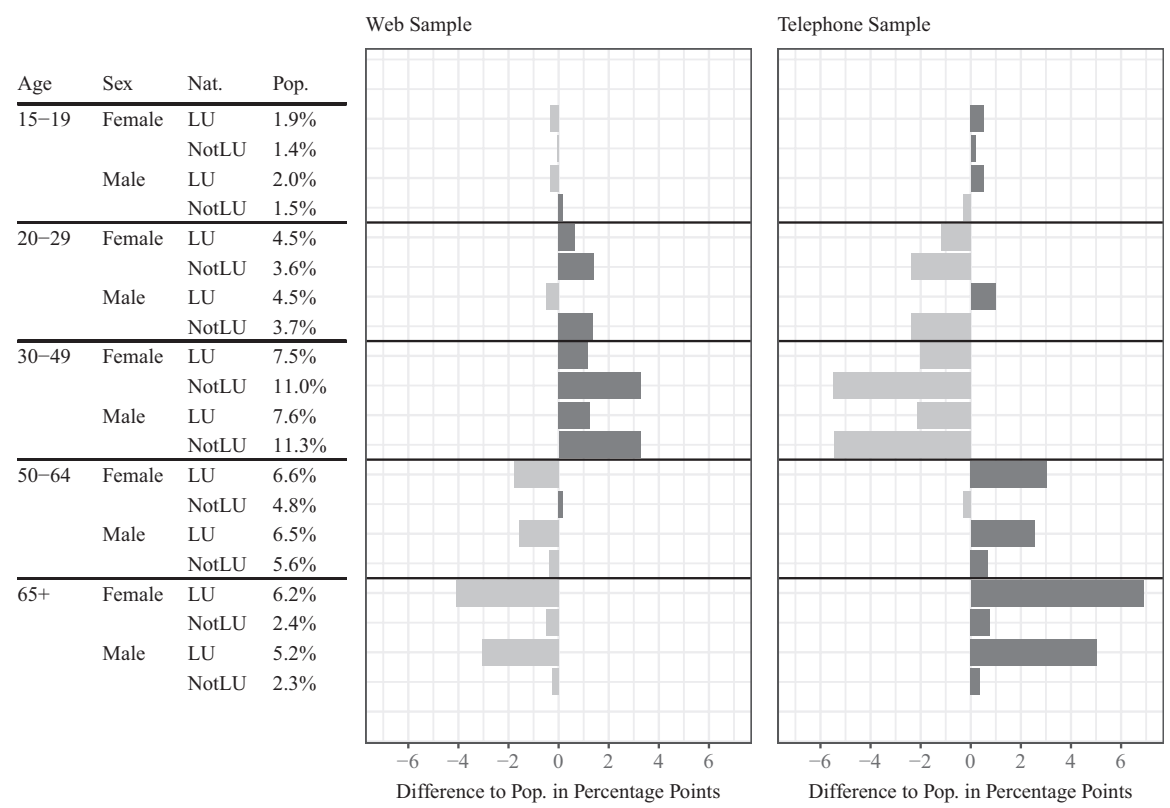

Fig. 1. Weighted cross tabulation of age, sex, and nationality for population, web, and telephone samples of the LFS 2017. Nat. = nationality; Pop. = population

between 20-49 are more often collected by web and age groups $50+$ are more often collected by telephone. Second, responses from Luxembourgish people are collected more often by telephone.

To base our evaluation of whether the Luxembourgish LFS data is affected by modespecific measurement bias on a generalizable and large enough sample, we combine all available data that was collected via mixed survey mode up to this point (i.e., 2015, 2016 and 2017). Of this data set, we use each household reference person with an age of 15-74 years, leading to a sample size of $n=57,566$. The final data consists of $60 \%$ web interviews, with varying ratios for the three different years (57\% in 2015 ; $65 \%$ in 2016 ; $59 \%$ in 2017).

For our investigation on mode-specific measurement bias, we use three different variables as outcomes: "Employment Status", "Wage Adequacy", and "Job Satisfaction". Employment Status is defined according to the guidelines of the ILO, International Labour Organisation (ICLS Resolution (19th) 2013) and is derived from three LFS variables (WSTATOR, AVAILABL, and SEEKWORK). Respondents are considered to be employed if they do any work for pay or profit, are not working but have a job from which they are absent, or are not working because of lay off. To further determine if respondents are non-labour (i.e., outside the labourforce) or unemployed according to the ILO definition, questions about whether a respondent is available to start working or is seeking employment are used. The two variables Wage Adequacy and Job Satisfaction are each collected with a single item, asking respondents to self-assess their attitude on a four-point rating scale.

We use the variables Employment Status, Wage Adequacy, and Job Satisfaction as outcomes for multiple reasons: First, we consider Employment Status as objective variable 
and Wage Adequacy as well as Job Satisfaction as subjective variables. All of the variables contain relatively sensitive information and are therefore more likely to be affected by measurement bias. Analysing these variables therefore allows comparisons of objective and subjective variables. Second, Employment Status is typically the most important variable of the LFS and, hence, the results for Employment Status have important implications for the Luxembourgish LFS as well as for the LFS of other countries. Third, Luxembourg collects Wage Adequacy and Job Satisfaction in addition to the mandatory LFS variables that are mainly objective. This provides us with the possibility to extend previous mixed mode literature of the field of labour force research, which focuses mainly on objective variables.

Wage Adequacy and Job Satisfaction are only available for a sub-sample of the LFS, since the two variables can only be collected for participants that are currently employed and they are only collected in the first LFS panel wave. Hence, we use two different samples for the following mixed mode analyses. Some auxiliary variables contain missing values due to item nonresponse. In order to base our mixed mode analyses on as much complete data as possible, we impute missing values prior to the mixed mode analyses. We conduct two separate single imputations for the Employment Status sample and the subsample of Wage Adequacy and Job Satisfaction. The two imputation models contain the matching variables defined in Subsection 5.1 as well as the outcome variables as predictors. Continuous variables are imputed by predictive mean matching (Van Buuren 2012; Meinfelder 2014; Morris et al. 2014) and categorical variables are imputed via multinomial logistic regression (Van Buuren 2012; Sentas and Angelis 2006). We conduct the imputation with the $\mathrm{R}$ programming language and the add-on package mice (Van Buuren 2012; Van Buuren and Groothuis-Oudshoorn 2011). The package uses a chained equations approach, which enables the user to impute all variables simultaneously (Azur et al. 2011).

\section{Method}

Since the present study relies on observational data, we need to control for differences between web and telephone samples that may be caused by nonresponse and coverage issues of the data (as illustrated in Figure 1) in order to enable a meaningful comparison of outcome variables. Subsection 4.1 discusses the matching method that we use for this purpose and Subsection 4.2 shows how we select auxiliary variables for the matching.

\subsection{Coarsened Exact Matching (CEM)}

CEM is a matching method introduced in the literature (Iacus et al. 2011; Iacus et al. 2012) that approximates randomized experiments by reducing dissimilarities between two samples (i.e., web and telephone samples). The advantage of CEM is that it ensures a similar distribution of auxiliary variables in the two samples and therefore allows the user to control ex-ante for potentially confounding influences of auxiliary variables (Iacus et al. 2012).

CEM coarsens auxiliary variables into meaningful groups, creates strata on the basis of these coarsened variables, and then only retains observations from strata that contain at least one observation from both samples. An important feature of CEM is that it allows to 
remove observations of both samples. When the number of web and telephone observations in a CEM stratum is unequal, weighting adjustments are made. We additionally multiply CEM weights by the original sample weights of the LFS to maintain representatives of the LFS sample (Riillo 2017). For the application of CEM, we use the R programming language and the add-on package cem (Iacus et al. 2018).

Subsequently, estimates are calculated using only the retained data and the original uncoarsened variables (Blackwell et al. 2009). If properly applied, CEM results in a subset that has similar characteristics not only on average, but on the whole distribution of observable variables. This approximates an experimental setting and therefore allows the comparison of estimates using the two matched samples. CEM-based estimates have powerful statistical properties and can outperform other matching procedures (Iacus et al. 2011).

It is important to note that CEM requires some (untestable) assumptions to be satisfied: the Conditional Independence Assumption (CIA), the Stable Unit Treatment Value Assumption (SUTVA), as well as the assumption of a common support condition. The CIA requires that common variables affecting treatment assignment and treatment-specific outcomes are observable. The SUTVA requires that the treatment applied to one unit does not affect the outcome of another unit. The common support condition requires that there is a positive probability of being both treated and untreated for each value of the matching variable. However, a major benefit of CEM over regression analysis is that it is nonparametric and, hence, the linearity assumption does not have to be considered (see Morgan and Winship (2007) for further details).

\subsection{Construction of CEM Models}

Constructing an appropriate matching model (i.e., a set of auxiliary variables that is used to match web and telephone samples) is an important requirement for the functionality of CEM (Iacus et al. 2012; Mercer et al. 2017). To produce unbiased estimates of a group effect (i.e., mode-specific measurement bias), all variables that affect the likelihood to be in a certain group (i.e., being surveyed by web or telephone) need to be included in the matching model (Imbens 2004; Rosenbaum and Rubin 1983). In addition, we include strong predictors for the outcomes in the matching models. Even though predictors for an outcome only affect the potential bias of CEM, if they are also related to the likelihood of being in a certain group, such predictors can decrease the variance of group effect estimates (Brookhart et al. 2006; Shrier et al. 2007; Stuart 2010). Furthermore, matching models should use as few variables as possible, since each additional variable reduces the chance of finding matching partners in both samples (Iacus et al. 2012).

To find the best matching model, we use a combination of automatic variable selection via random forest and the literature to find relevant auxiliary variables. As a first step, we conduct an automatic variable selection via random forest. Random forest is a machine learning method that can be used to rank a set of $K$ auxiliary variables $X_{k}$ with $k=1,2, \ldots$, $K$ by their importance for an outcome $Y$, providing the user with the ability to select the most important auxiliary variables for $Y$ (Breiman 2001). We apply random forest based on the $\mathrm{R}$ programming language and the add-on package randomForest (Breiman et al. 2018; Liaw and Wiener 2002). We conduct two separate random forest runs, each with a 
different $Y$ : First, we apply random forest with a binary variable for survey mode as $Y$ (i.e., web $=0$; telephone $=1$ ) in order to find variables that explain why certain participants are more likely to be in the web or telephone sample. Second, we determine strong predictors for our outcome variables Employment Status, Wage Adequacy, and Job Satisfaction by specifying each of these outcome variables as $Y$ within a random forest procedure. We then combine the automatically selected variables of both runs into one pool of variables.

Subsequently, we conduct a literature-based evaluation of whether the automatically selected pool of variables includes all variables that were identified as important predictors in previous research. To ensure the inclusion of important predictors for survey mode, we compare our automatically selected pool of variables with research about the characteristics of respondents in web and telephone samples (Körner and Liersch 2014; Sarracino et al. 2017; Schouten and Van der Laan 2014). We also examine research about the probability of appearing in the telephone book, the main factor influencing why participants are assigned to one of the two survey modes in the Luxembourgish LFS (Grande and Taylor 2010; Lipps et al. 2013). In addition, we examined the literature concerning modelling of employment variables similar to the ones in our study to ascertain the predictive power of the variables selected by the random forest (Bos et al. 2009; Garrouste and Rodrigues 2014; Sousa-Poza and Sousa-Poza 2000; Ting 1997; Vila and Garcia-Mora 2005). According to these literature-driven evaluations, we adjust the pool of automatically selected variables and define final matching models for the CEM (as discussed in Subsection 5.1). Furthermore, we use previous research literature to assess whether the Luxembourgish LFS is lacking any important predictors and evaluate the potential impact of such non-observed predictors on our results (as shown in Subsection $6.2)$.

\section{Results}

The results of the present study are split into two parts. Subsection 5.1 discusses the matching models. We compare sample compositions before and after CEM, which enables an evaluation of whether CEM successfully balanced web and telephone samples according to the matching variables. Subsection 5.2 presents the major results of the present study by comparing the outcome variables before and after CEM. Results of these comparisons provide the basis for a discussion of the magnitude of mode-specific measurement bias in objective and subjective variables.

\subsection{Sample Compositions Pre- and Post-CEM}

As result of the variable selection, we use two different matching models: One for Employment Status and another one for the two subjective variables, Wage Adequacy and Job Satisfaction. We use different matching models for two reasons. First, the samples for Employment Status and the two subjective variables differ (as discussed in Section 3). The different samples also lead to different sets of potential matching variables. For instance, "Nomenclature of Economic Activities" (NACE), "International Standard Classification of Occupations" (ISCO), and salary can be collected only for the same sub-sample as Wage Adequacy and Job Satisfaction. These variables can therefore not be used as matching variables for Employment Status. 
Second, potential matching variables have a different importance for Employment Status and the subjective variables, respectively. As discussed in Subsection 4.2. matching variables should be related to survey mode as well as to the outcome. While predictors for survey mode remain the same for all matching models, different variables are relevant for the outcomes. For instance, the data collection month was identified to be a strong predictor for Employment Status (since the variable explains seasonality on the labour force market), but not for Wage Adequacy and Job Satisfaction. Data collection month was therefore only included as a matching variable for Employment Status. In addition, the larger sample size for Employment Status enables a more complex matching model, since it is easier to find finely defined matches when the sample size is larger (i.e. CEM reduces the sample size less). We therefore selected a slightly larger matching model for Employment Status.

For Employment Status the selected matching variables are age, sex, nationality, country of birth, "International Standard Classification of Education" (ISCED), marital status, questionnaire language, collection month, collection year, and panel wave. For both Wage Adequacy and Job Satisfaction, we use age, sex, nationality, ISCED, NACE, ISCO, net salary, questionnaire language, and collection year. The predictive power of collection year and panel wave is relatively small, but the two variables were added to prevent a match between the same participant collected in different years or waves. Detailed information about the LFS variable definitions is available in Eurostat (2018a). Tables 6 and 7 in the online Supplemental Material show precise numbers for differences before and after matching.

We coarsen age into 15 equally wide intervals; nationality into Luxembourgish and non-Luxembourgish; ISCED into three groups representing low, middle, and high education; marital status into three groups with widowed and divorced combined into one group; NACE according to the Eurostat high-tech aggregation by NACE Rev.2 (Eurostat 2018b) into knowledge-intense services (KIS), less knowledge-intensive services (LKIS), manufacturing industries (Manuf), as well as into a group called other, which consists of NACE groups that are not covered by the applied Eurostat aggregation; ISCO into four groups; and net salary into five income groups, as well as into a group for non-employees.

Before CEM, almost all estimates for sub-groups differ significantly between web and telephone samples, which shows that it would not be possible to evaluate mode-specific measurement bias based on the unmatched data. The only non-significant differences were observed for collection month August for the Employment Status sample and the NACE category KIS in the case of the sub-sample of Wage Adequacy and Job Satisfaction. After CEM, in contrast, none of the differences are statistically significant, indicating that the imbalance between matched web and telephone samples is sufficiently reduced according to the coarsened sample characteristics and, thus, enabling an evaluation of mode-specific measurement bias.

Iacus et al. (2011) introduced the global LI imbalance measure (ranging from 0 to 1 with $0=$ perfect balance and $1=$ maximum imbalance), which helps to evaluate whether CEM balanced the web and telephone samples successfully. In the present study, we measure an imbalance of $L 1=0.95$ for the Employment Status sample before applying CEM and $L 1=0.71$ after CEM. For the sub-sample of Wage Adequacy and Job Satisfaction we measure $L 1=0.97$ before CEM and $L 1=0.85$ after CEM. Even though some imbalance 
remains, the L1 measures suggest that CEM considerably reduced the imbalance between web and telephone samples. We address the impact of remaining imbalance in Subsection 6.1 via multinomial logit regression.

\subsection{Outcome Variables Pre- and Post-CEM}

To evaluate whether the outcomes for Employment Status, Wage Adequacy and Job Satisfaction are affected by measurement bias, we compare the proportions of outcome categories in the matched web and telephone samples. Figure 2 illustrates differences for web and telephone samples before and after applying CEM for Employment Status by displaying proportions for the three Employment Status categories employed, unemployed, and non-labour. Weighted web and telephone samples before matching are represented by black and dark grey bars. Weighted web and telephone samples post matching are shown in medium grey and light grey.

Proportions of web and telephone samples before matching are very different within the categories. In comparison to the telephone sample, web participants are much more often employed and are slightly more often unemployed. In contrast, web participants have a non-labour status less often than participants of the telephone sample.

After CEM, however, differences between web and telephone samples within the three outcome categories are not statistically significant (see Table 1 for precise numbers), indicating that differences before matching are exclusively due to sample compositions of web and telephone data. The results therefore suggest that Employment Status is not affected by mode-specific measurement bias.

Figure 3 visualizes differences before and after matching for the two outcomes Wage Adequacy and Job Satisfaction. Both variables consist of the categories strongly agree, agree, disagree, and strongly disagree, with strongly agree for people with the highest

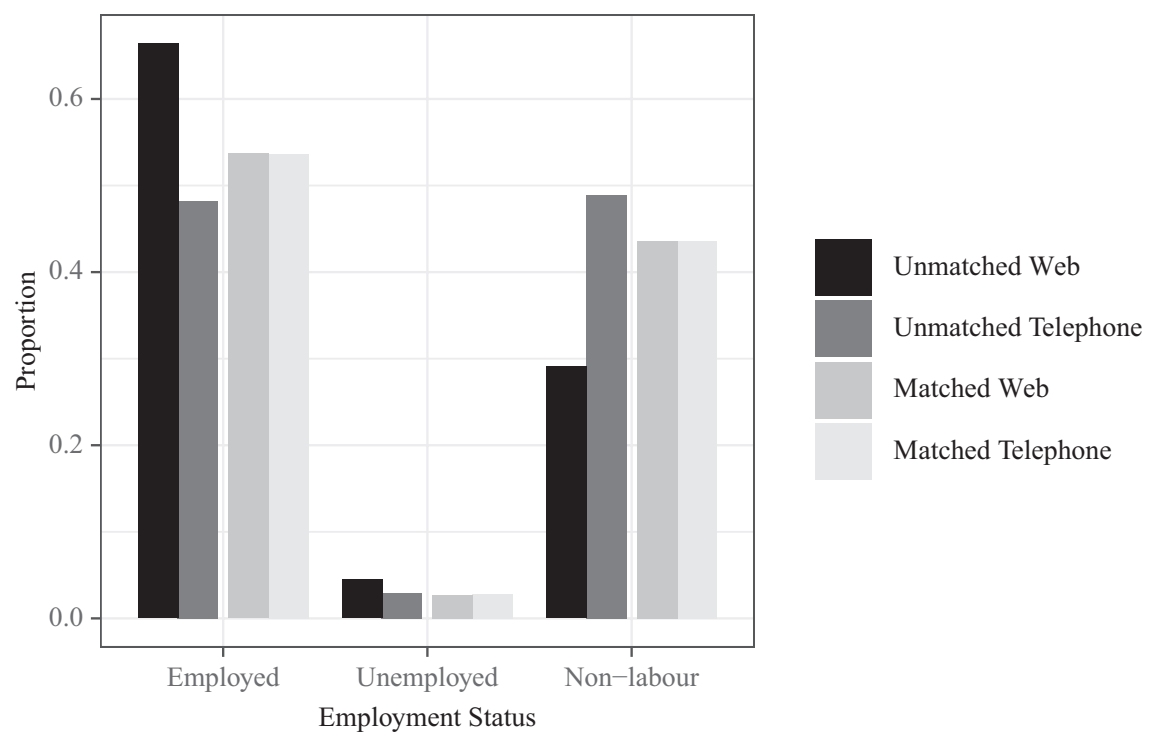

Fig. 2. Mode-specific measurement bias on employment status. 
Wage Adequacy or Job Satisfaction, respectively. As in Figure 2, web and telephone pre CEM are represented by the colours black and dark grey and web and telephone post CEM are represented by medium and light grey.

Before CEM, web participants perceive their salary less adequate than telephone respondents. Web participants are less likely than the telephone participants to select the categories strongly agree or agree and more likely to select the categories disagree or strongly disagree. Similar observations can be made for the variable Job Satisfaction. Unmatched web participants select the category strongly agree less often than telephone participants. The categories agree, disagree, and strongly disagree, in contrast, are selected more often by unmatched web participants. To both of these variables, unmatched telephone participants give more positive responses. Notably, systematic differences between web and telephone samples remain after CEM. Telephone participants still have a higher probability to select positive categories in the matched data.

Table 1 shows web and telephone proportions, unconditional mean, absolute differences, and relative differences in percent. In case of the variable Wage Adequacy, we observe differences between matched web and telephone samples are as low as 2.2 percentage points for the category strongly disagree and as high as 8.2 percentage points for the category disagree. We note that on average $19.9 \%$ of the matched web and telephone respondents answer disagree (unconditional mean). The likelihood to respond disagree is therefore decreased by $35.2 \%$ when a response is collected by telephone instead of web.

The differences between matched web and telephone samples of the variable Job Satisfaction range between 1.7 percentage points for strongly disagree and 11.9 percentage points for strongly agree. The unconditional mean of the category strongly agree is $33.0 \%$, that is, the likelihood of reporting strongly agree is increased by $42.3 \%$ when a respondent is asked by telephone instead of web. The results therefore indicate that the two subjective variables Wage Adequacy and Job Satisfaction are substantially affected by modespecific measurement bias.

Overall, the results are in line with our expectations. While pre-CEM differences of the objective variable Employment Status could be explained by auxiliary variables, the
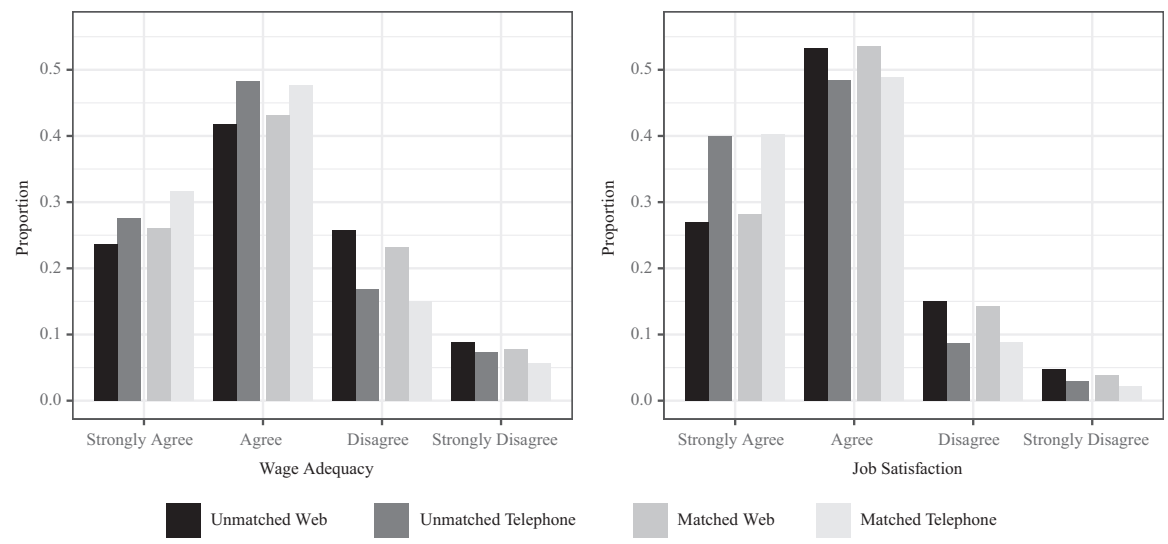

Fig. 3. Mode-specific measurement bias on wage adequacy and job satisfaction. 
Table 1. Web, and telephone proportions of each category, unconditional mean, absolute differences (i.e., telephone - web) and relative differences (i.e., (telephone - web)/web). Values are reported in percent. Prop. $=$ proportion; Diff $=$ difference.

\begin{tabular}{lrrrrr}
\hline Variable & $\begin{array}{c}\text { Web } \\
\text { prop. }\end{array}$ & $\begin{array}{c}\text { Phone } \\
\text { prop. }\end{array}$ & $\begin{array}{c}\text { Mean } \\
\text { prop. }\end{array}$ & $\begin{array}{c}\text { Absolute } \\
\text { diff. }\end{array}$ & $\begin{array}{r}\text { Relative } \\
\text { diff. }\end{array}$ \\
\hline Employment status & & & & & \\
$\quad$ Employed & 53.7 & 53.6 & 53.6 & 0.1 & -0.1 \\
$\quad$ Unemployed & 2.7 & 2.8 & 2.8 & 0.0 & 1.8 \\
$\quad$ Non-labour & 43.6 & 43.6 & 43.6 & 0.0 & 0.1 \\
Wage adequacy & & & & & \\
$\quad$ Strongly agree & 26.0 & 31.7 & 28.3 & 5.8 & 22.2 \\
Agree & 43.1 & 47.6 & 44.9 & 4.6 & 10.7 \\
Disagree & 23.2 & 15.0 & 19.9 & -8.2 & -35.2 \\
$\quad$ Strongly disagree & 7.8 & 5.6 & 6.9 & -2.2 & -27.9 \\
Job satisfaction & & & & & \\
$\quad$ Strongly agree & 28.2 & 40.2 & 33.0 & 11.9 & 42.3 \\
Agree & 53.6 & 48.9 & 51.7 & -4.7 & -8.8 \\
$\quad$ Disagree & 14.3 & 8.8 & 12.0 & -5.5 & -38.7 \\
$\quad$ Strongly disagree & 3.9 & 2.2 & 3.3 & -1.7 & -43.2 \\
\hline
\end{tabular}

subjective variables Wage Adequacy and Job Satisfaction still show differences after CEM. The results therefore suggest that objective variables are less affected by modespecific measurement bias than subjective variables.

\section{Supplementary Analyses}

We conduct several supplementary analyses to assess the validity and stability of our results (Athey and Imbens 2017). Subsection 6.1 assesses whether the CEM results are confirmed by a multinomial logit, which estimates mode-specific measurement bias using uncoarsened auxiliary variables. Subsection 6.2 discusses the issue of unobserved confounders and examines the potential effect of such confounders on the results of the present study.

\subsection{Multinomial Logit}

In addition, we analyzed data from the matched participants using multinomial logit, which is suited to model categorical variables such as our outcomes. As a robustness check, we also implemented an ordered logit model. The model imposed the same coefficients of the outcomes for all outcome categories. This assumption, known in the literature as parallel regression assumption (Long and Freese 2006), is violated in our data. With multinomial logit, we estimate simultaneously the associations between survey mode and all categories of the outcomes, accounting for auxiliary variables that explain the outcomes. Hereby, we use auxiliary variables in their uncoarsened format and, hence, model imbalances that remain after CEM. Implementing multinomial logit on CEM retained data enables the estimation of the impact of survey mode on the outcomes, on a sample of observations that resemble experimental data. 
Marginal effects of survey mode on each category, standard errors, and significance levels are illustrated in Table 2 for Employment Status, in Table 3 for Wage Adequacy, and in Table 4 for Job Satisfaction. In each model, we control for all matching variables in their uncoarsened format. Multinomial logit estimates - Tables 8, 9, and 10 - and average marginal effects - Tables 11, 12, and 13 - of the complete models are reported in the online Supplemental Material. Table 2 shows that survey mode does not have a significant impact on any of the three Employment Status categories, since none of the marginal effects is significantly different from zero. In contrast, Table 3 and 4 show that survey mode has a significant effect on all of the categories of Wage Adequacy and Job Satisfaction. The directions of the coefficients are in line with Figure 3. Telephone survey mode leads to a higher likelihood of responding strongly agree and agree to the variable Wage Adequacy as well as strongly agree to the variable Job Satisfaction; Web survey mode makes it more likely to respond disagree and strongly disagree to the variable Wage Adequacy as well as agree, disagree, and strongly disagree to the variable Job Satisfaction.

Table 2. Telephone survey mode as a predictor of employment status categories. Average marginal effects are computed via multinomial logit.

\begin{tabular}{lccc}
\hline & Employed & Unemployed & Non-labour \\
\hline Tel. mode & $-0.00316(0.009)$ & $0.000860(0.003)$ & $0.00230(0.009)$ \\
\hline Sample size & 12,971 & 12,971 & 12,971 \\
\hline $\begin{array}{l}\text { Standard errors in parentheses } \\
\bullet p<0.10, * p<0.05, * * p<0.01\end{array}$ & &
\end{tabular}

Table 3. Telephone survey mode as a predictor of wage adequacy categories. Average marginal effects are computed via multinomial logit.

\begin{tabular}{lcccc}
\hline & Strongly agree & Agree & Disagree & Strongly disagree \\
\hline Tel. mode & $\begin{array}{c}0.0562^{* *} \\
(0.017)\end{array}$ & $\begin{array}{c}0.0430^{*} \\
(0.019)\end{array}$ & $\begin{array}{c}-0.0784^{* *} \\
(0.015)\end{array}$ & $\begin{array}{c}-0.0208^{*} \\
(0.010)\end{array}$ \\
\hline Sample size & 4,015 & 4,015 & 4,015 & 4,015 \\
\hline
\end{tabular}

Standard errors in parentheses

- $p<0.10, * p<0.05, * * p<0.01$

Table 4. Telephone survey mode as a predictor of job satisfaction categories. Average marginal effects are computed via multinomial logit.

\begin{tabular}{lcccc}
\hline & Strongly agree & Agree & Disagree & Strongly disagree \\
\hline Tel. mode & $\begin{array}{c}0.122^{* *} \\
(0.018)\end{array}$ & $\begin{array}{c}-0.0507^{* *} \\
(0.019)\end{array}$ & $\begin{array}{c}-0.0541^{* *} \\
(0.012)\end{array}$ & $\begin{array}{c}-0.0169^{* *} \\
(0.006)\end{array}$ \\
\hline Sample size & 4,015 & 4,015 & 4,015 & 4,015 \\
\hline
\end{tabular}

Standard errors in parentheses

- $p<0.10, * p<0.05, * * p<0.01$ 
An important question for practitioners is the strength of the survey modes' impact on collecting subjective variables. As shown in Table 3, for Wage Adequacy we measure the strongest impact of survey mode on the category disagree - telephone respondents are 7.8 percentage points less likely to respond disagree. In comparison, the strongest predictor for this category is to have a doctoral degree instead of primary education, which makes it 21.2 percentage points more likely to respond disagree (see Table 12).

The impact of survey mode appears to be even stronger for the category strongly agree of the variable Job Satisfaction. Table 4 shows that telephone respondents are 12.2 percentage points more likely than web respondents to report strongly agree. The most influential predictor of Job Satisfaction is being a manager instead of having an elementary occupation, which increases the probability of strongly agree by 24.7 percentage points (see Table 13). Overall, the multinomial logit analysis confirms the findings of Subsection 5.2 , suggesting that survey mode has a non-negligible impact only on subjective questions.

\subsection{Unobserved Confounders}

A general issue of matching methods such as CEM is the potential existence of unobserved confounders, that is, the used data set lacks important auxiliary variables that affect survey mode and outcome variables (Mercer et al. 2017; Rosenbaum 2005). Not accounting for unobserved confounders might lead to insufficient matching and therefore to flawed mixed mode conclusions.

As described in Subsection 4.2, we evaluated based on previous research literature whether the Luxembourgish LFS data might lack important variables for our matching models. According to this literature, the most important predictors for survey mode as well as for the outcome Employment Status are considered in our analyses. However, the risk of lacking predictors for the subjective outcomes Wage Adequacy and Job Satisfaction (e.g., having an exhausting job or relations with colleagues) seems to be higher. It is unlikely that these predictors simultaneously have a strong impact on survey mode, that is, it is unlikely that these variables cause bias in the results of the present study. However, the absence of these variables might still reduce the quality of CEM and, furthermore, we might not have spotted all important unobserved confounders in previous literature. To evaluate the potential impact of unobserved confounders on our results we conduct several analyses.

Three types of analysis can usually be conducted to assess the effect of unobserved confounders: falsification tests; robustness checks; and sensitivity analyses (Athey and Imbens 2017). In the present study, we do not apply falsification tests due to data limitations. Falsification tests usually check for placebo effects by replacing the outcome under study with a pseudo-outcome that is theoretically known to be unaffected by the group. The researcher then examines whether the chosen method (i.e., CEM) leads to effects different from zero on the pseudo-outcome, whereby significant effects indicate a higher risk of bias due to unobserved confounders. Variables that are used as pseudooutcomes are typically related to the outcome under study, for example variables with similar content or the lagged outcome (Athey and Imbens 2017). Unfortunately, such variables are not available in our data. We cannot doubtlessly know the association of potential pseudo-outcomes with survey mode and we also do not have lagged data of our 
entire sample before the implementation of the mixed mode survey design. Instead, we focus on robustness checks and sensitivity analyses in order to evaluate the potential risk of unobserved confounders.

\subsubsection{Robustness Checks}

Robustness checks assess whether the results of a study change when they are determined by an alternative measurement method. To check the robustness of our group effect estimates of the present study, we assess whether our estimates change when we measure the group effects using different matching models. To do this, we successively add further variables to the matching models and examine whether the estimates converge to a certain value. We consider our estimates to be robust if they no longer change substantially above a certain level of complexity.

Figure 4 illustrates how the differences of web and telephone sample proportions change, when variables are successively added to the matching models. Employment Status is visualized on the left side; Wage Adequacy is illustrated in the middle; and Job Satisfaction is shown on the right side. Each category of each outcome variable is displayed separately, that is, employed, unemployed, and non-labour for Employment Status and strongly agree, agree, disagree, and strongly disagree for Wage Adequacy and Job Satisfaction. The first points on the left side of each of the three graphics reflects the differences of web and telephone samples before matching (i.e. the black bars minus the dark grey bars of Figure 2 and Figure 3, respectively).

Illustrated by the second points of each graphic, we start matching according to collection year and panel wave in order to ensure that a participant cannot be matched to itself (in case of Wage Adequacy and Job Satisfaction only for collection year, since the

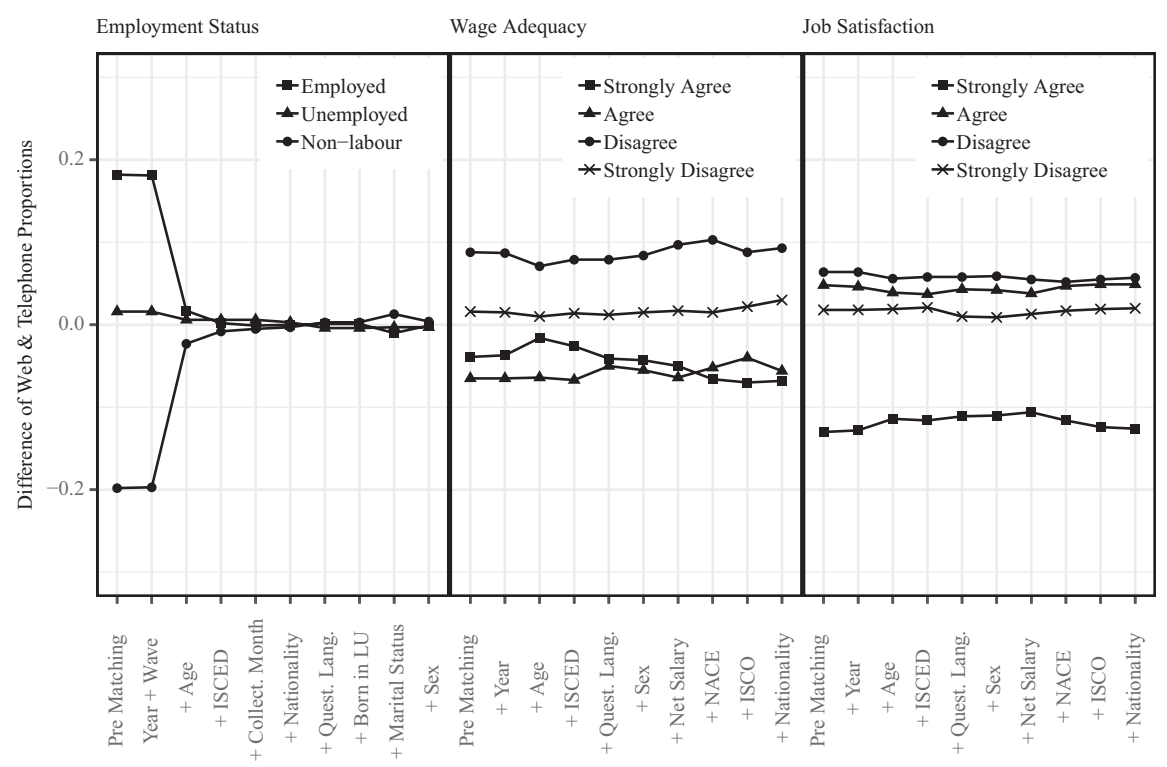

Fig. 4. Robustness checks for matching models. 
variables are collected only in the first wave of the Luxembourgish LFS). Afterwards, we successively add further variables to the matching models, whereby the variables are approximately ordered by their impact on the difference of matched web and telephone samples. Each point of each line reflects the differences between web and telephone samples based on the given matching model. The last points of each graphic correspond to the final matching model that was actually used for the analysis of the present study (i.e. the medium grey bars minus the light grey bars of Figure 2 and Figure 3, respectively).

The differences between web and telephone samples of the variable Employment Status are reduced drastically after the addition of the most important variables age, ISCED, and collection month. Adding more variables, however, does not substantially change the differences any further - the differences of all three categories converge close to zero. For Wage Adequacy and Job Satisfaction, the matching variables cause only small changes, leading to a similar level of differences across the matching models. In summary, the addition of further variables to the three matching models does not seem to change the differences between web and telephone samples. All of the outcomes therefore seem to be robust to alternative ways of measuring the group effect, which supports the assumption that the analysis is not substantially biased by unobserved confounders.

\subsubsection{Sensitivity Analyses}

Sensitivity analyses measure how important unobserved confounders need to be in order to sufficiently change the group effect estimate. As suggested by VanderWeele and Ding (2017), we measure sensitivity based on the E-value. The E-value is defined as the minimum association on the risk ratio scale of an unobserved variable with both the group assignment (i.e. the survey mode) and the outcome in order to nullify an observed association between group assignment and outcome, conditional on the observed variables. The E-value has a range from 1 to infinity, with smaller values implying that only a smaller effect of unobserved confounders would be needed to nullify an observed association. The higher an E-value, the less sensitive an analysis is to unobserved confounders.

To determine the E-values, we use the function evalues. $O R$ of the $\mathrm{R}$ add-on package EValue, which calculates E-values based on odds ratios (Mathur et al. 2018). Table 5 shows odds ratios for the categories of the three outcomes; E-values for the odds ratio point estimates; E-values of confidence interval limits closer to the null $(p<.05)$; as well as an identifier for rare outcomes. Following the suggestion of VanderWeele and Ding (2017), we specify outcome variables as rare when the outcome occurs in less than $15 \%$ of the cases.

E-values for the Employment Status categories are close to 1 with lower or upper confidence intervals equal to 1 , indicating that no additional confounders would be needed to nullify the observed effect. As shown in Section 5 of the present study, Employment Status is not significantly affected by survey mode, leading to an odds ratio close to 1 . The low E-values are therefore in line with our expectations. The E-values are substantially higher for the two subjective outcomes Wage Adequacy and Job Satisfaction. In case of Wage Adequacy, the E-Values are 1.6 for strongly agree, 1.4 for agree, 2.0 for disagree, and 2.2 for strongly disagree. In case of Job Satisfaction, the E-Values are 2.0 for strongly agree, 1.4 for agree, 2.9 for disagree, and 3.1 for strongly disagree. The associations of 
unobserved confounders with both survey mode and the subjective outcomes would need to have at least these sizes to potentially nullify the observed associations.

To address the special situation of Employment Status, where the observed associations are not statistically different from null, we evaluate how strong an unobserved association would need to be to potentially move these near-null associations to meaningful levels (see VanderWeele and Ding (2017) for detailed guidelines on how to evaluate E-values for non-null hypotheses). Figure 5 illustrates E-values for increasing tested odds ratios, indicating how strong an unobserved association would need to be to shift the observed odds ratio to a different level. As already indicated by Table 5, the minimum E-value for the three Employment Status categories is close to a tested odds ratio of 1 . However, when the tested odds ratio is set closer to 0 or higher than 1, the E-value continuously increases. For instance, an unobserved association with survey mode and the Employment Status

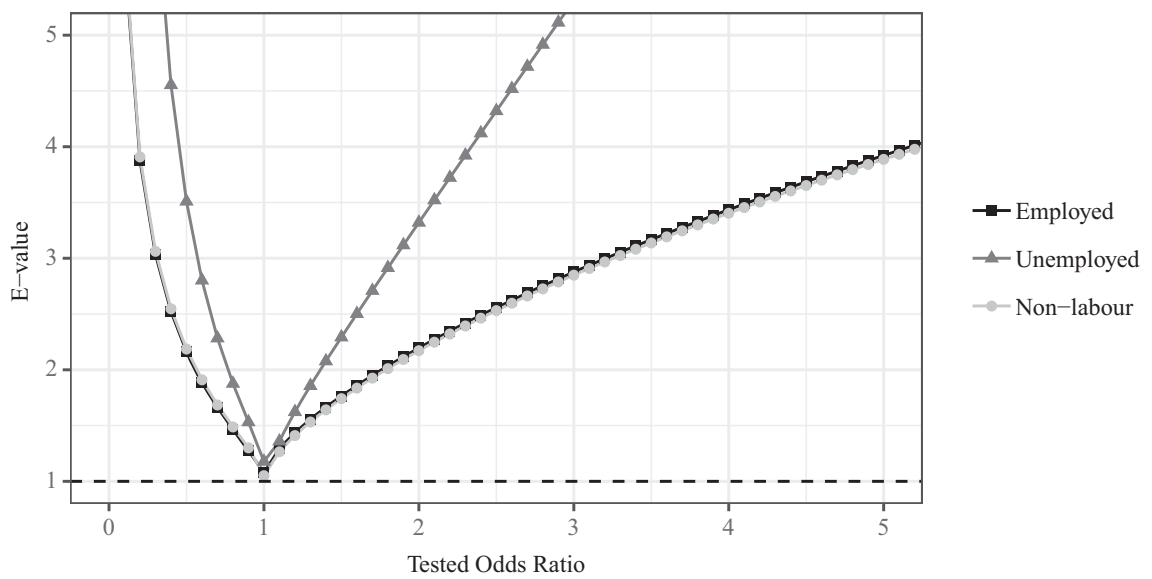

Fig. 5. E-values for employment status with increasing tested odds ratio.

Table 5. E-value sensitivity analyses for employment status, wage adequacy, and job satisfaction.

\begin{tabular}{lccccc}
\hline Variable & Odds ratio & E-value & Lower CI & Upper CI & Rare \\
\hline $\begin{array}{l}\text { Employment status } \\
\quad \text { Employed }\end{array}$ & 1.0 & 1.1 & - & & \\
$\quad$ Unemployed & 1.0 & 1.2 & 1.0 & - & Yes \\
$\quad$ Non-labour & 1.0 & 1.1 & 1.0 & - & No \\
Wage adequacy & & & & & \\
$\quad$ Strongly agree & 1.3 & 1.6 & 1.3 & - & No \\
$\quad$ Agree & 1.2 & 1.4 & 1.1 & - & No \\
Disagree & 0.6 & 2.0 & - & 1.7 & No \\
$\quad$ Strongly disagree & 0.7 & 2.2 & - & 1.2 & Yes \\
Job satisfaction & & & & & \\
$\quad$ Strongly agree & 1.8 & 2.0 & 1.7 & - & No \\
$\quad$ Agree & 0.8 & 1.4 & - & 1.2 & No \\
$\quad$ Disagree & 0.6 & 2.9 & - & 2.1 & Yes \\
$\quad$ Strongly disagree & 0.5 & 3.1 & - & 1.6 & Yes \\
\hline
\end{tabular}


categories would need to be higher than two in order to move the observed odds ratio to 0.5 or 2.0, respectively.

With respect to the content of our group and outcome variables, the magnitude of the computed E-values supports the results of the present study. Associations higher than two or three occasionally occur, but are not very common (VanderWeele and Ding 2017). An unobserved confounder with such associations to both survey mode and our outcomes is even less likely. Even though some categories of the subjective outcomes are relatively sensitive to unobserved confounders (especially the category agree of both Wage Adequacy and Job Satisfaction), it is unlikely that all observed associations could be nullified. In case of the outcome variable Employment Status, it seems unlikely that an unobserved confounder could be capable of moving the observed non-significant associations to a meaningful level.

\section{Summary and Outlook}

High standards during the data collection process are a necessary precondition in order to produce high quality survey data. Mixed survey modes lead to additional challenges during collection and analysis of survey data, since the way data is collected may affect the response of survey participants. Depending on the topic of a survey and the content of a specific variable, research found different magnitudes of mode-specific measurement bias, ranging from non-negligible differences in participants' response behavior to no systematic measurement effect at all.

We contributed to this research by investigating on objective and subjective variables collected in the Luxembourgish LFS, one of the largest surveys on employment related variables. We reduced differences in the observed composition of web and telephone samples using CEM in order to simulate a random experiment and separated modespecific measurement bias from mode-specific coverage and mode-specific. nonresponse rates. We applied CEM to the objective variable Employment Status and to the two subjective variables Wage Adequacy and Job Satisfaction.

The results of the present study show that mode-specific measurement bias has a different magnitude depending of the content of a specific variable. We found no significant measurement bias for Employment Status, but substantial effects for the two subjective variables Wage Adequacy and Job Satisfaction. Results therefore suggest that measurement bias is less problematic for objective than for subjective variables. We assume that the presence of an interviewer affects participants' response behavior for example because of social desirability effects. The main conclusion of the present study is that even if there is no difference in terms of coverage and nonresponse, subjective questions can convey substantially different results if collected by web or by telephone.

Especially the results for Employment Status have important implications not only for Luxembourgish data, but for the LFS of all countries in general. The high degree of formalization according to the ILO guidelines and the objectiveness of Employment Status provide international comparability that is not affected by collection mode. The subjective variables Wage Adequacy and Job Satisfaction only appear in the Luxemburgish LFS and therefore are not as important for the LFS of other countries as the variable Employment Status. However, the results for these variables generally show that data collection needs 
to be done with care, when collecting subjective variables. If the potentially diverging risk of mode-specific measurement bias in objective and subjective variables is disregarded, the results of mixed mode surveys might be misleading.

Our research has some limitations. We conducted our analysis based on observational data and balanced the data after collection via CEM, leading to the risk that the matched data might not be sufficiently balanced. There are two major risks: First, the selection and/or the coarsening of matching variables might be flawed. We therefore used a multinomial logit to evaluate whether a model with uncoarsened variables leads to different results. Second, important unobserved confounders for which we cannot account during the CEM process may exist. To evaluate this risk, we applied robustness checks and E-value sensitivity analyses. Overall, the supplementary analyses support the findings of the present study. The multinomial logit confirmed our results and based on the robustness checks and the E-value sensitivity analyses the risk of unobserved confounders that could explain away all the measured effects seems to be relatively low. However, in the future, researchers might conduct random experiments in order to eliminate any remaining doubts.

The difference between the two samples that we used for Employment Status and the two variables Wage Adequacy and Job Satisfaction is another limitation of the present study. Since Wage Adequacy and Job Satisfaction can only be collected for employed participants, our analyses reflect two populations. A related topic is the question of whether the observed differences between objective and subjective variables systematically appear in other variables. Even though the present study provides a good basis for the comparison of objective and subjective employment variables, a future field of research might be the analysis of additional objective and subjective variables that can be collected for the same population. This includes further investigations on employment related variables as well as variables of other research fields.

Research also needs to put more focus on the question of which mode leads to less biased results. The results of the present study provide a basis for this research by showing evidence that the magnitude of mode-specific measurement bias strongly depends on the content of a variable. We evaluated whether measurement bias exists and how large the differences between web and telephone data are. However, based on the present study it is not possible to judge which survey mode leads to results that are closer to the true value of the target population. Further research is also needed to develop an optimal procedure to correct for mode-specific measurement bias. Meanwhile, practitioners should carefully evaluate the content of a survey and its variables when choosing the best mode to conduct a survey.

\section{References}

Aggarwal, V.A., and D.H. Hsu. 2013. "Entrepreneurial exits and innovation." Management Science, 60(4): 867-887. DOI: https://doi.org/10.1287/mnsc.2013.1801. Athey, S., and G.W. Imbens. 2017. "The state of applied econometrics: Causality and policy evaluation.” Journal of Economic Perspectives, 31(2): 3-32. DOI: http: dx.doi. org/10.1257/jep.31.2.3. 
Azur, M.J., E.A. Stuart, C. Frangakis, and P.J. Leaf. 2011. "Multiple imputation by chained equations: What is it and how does it work?" International Journal of Methods in Psychiatric Research, 20(1): 40-49. DOI: https://doi.org/10.1002/mpr.329.

Bell, J., Huber, J., and W.K. Viscusi. 2011. "Survey mode effects on valuation of environmental goods." International Journal of Environmental Research and Public Health, 8(4): 1222-1243. DOI: https://doi.org/10.3390/ijerph8041222.

Bianchi, A., Biffgnandi, S., and P. Lynn. 2017. "Web-face-to-face mixed-mode design in a longitudinal survey: Effects on participation rates, sample composition, and costs." Journal of Official Statistics, 33(2): 385-408. DOI: 10.1515/jos-2017-0019.

Blackwell, M., S. Iacus, G. King, and G. Porro. 2009. "CEM: Coarsened Exact Matching in Stata." The Stata Journal, 9: 524-546. DOI: https://doi.org/10.1177/1536867X0900 900402.

Blanke, K., and A. Luiten. 2014. Query on Data Collection for Social Surveys. ESSnet Project "Data Collection for Social Surveys using Multiple Modes". Available at: http: //ec.europa.eu/eurostat/cros/system/files/Query_report_DCSS.pdf_en (accessed October 2019).

Bos, J.T., N.C.G.M. Donders, K.M. Bouwman-Brouwer, and J.W.J.V. der Gulden. 2009. "Work characteristics and determinants of job satisfaction in four age groups: university employees' point of view." International Archives of Occupational and Environmental Health, 82(10): 1249-1259. DOI: https://doi.org/10.1007/s00420-009-0451-4.

Breiman, L. 2001. "Random Forests". Machine Learning, 45(1): 5-32. DOI: https://doi.org/10.1023/A:1010933404324.

Breiman, L., A. Cutler, A. Liaw, and M. Wiener. 2018. Package randomForest. Available at: https://cran.r-project.org/web/packages/randomForest/randomForest.pdf (accessed February 2021).

Brookhart, M.A., S. Schneeweiss, K.J. Rothman, R.J. Glynn, J. Avorn, and T. Stärmer. 2006. "Variable selection for propensity score models." American Journal of Epidemiology, 163(12): 1149-1156. DOI: https://doi.org/10.1093/aje/kwj149.

Buelens, B., and J.A. van den Brakel. 2015. "Measurement Error Calibration in Mixedmode Sample Surveys." Sociological Methods and Research, 44(3): 391-426. DOI: https://doi.org/10.1177/0049124114532444.

Couper, M.P. 2011. "The Future of Modes of Data Collection." Public Opinion Quarterly (5): 889-908. DOI: https://doi.org/10.1093/poq/nfr046.

De Leeuw, E.D. 2005. “To Mix or Not to Mix Data Collection Modes in Surveys.” Journal of Official Statistics, 21(2): 233-255. Available at: https://www.scb.se/contentassets/ ca21 efb41fee47d293bbee5bf7be7fb3/to-mix-or-not-to-mix-data-collection-modes-insurveys.pdf. (accessed December 2020).

De Leeuw, E.D. 2018. "Mixed-mode: Past, present, and future." Survey Research Methods, 12(2): 75-89. DOI: https://doi.org/10.18148/srm/2018.v12i2.7402.

Dillman, D.A. 2017. "The promise and challenge of pushing respondents to the web in mixed-mode surveys." Survey Methodology, 43(1): 3-30. Available at: http://www .statcan.gc.ca/pub/12-001-x/2017001/article/14836-eng.htm (accessed February 2021).

Dillman, D.A., and L.M. Christian. 2005. "Survey Mode as a Source of Instability in Responses across Surveys." Field Methods, 17(1): 30-52. DOI: https://doi.org/10.1177/ 1525822X04269550. 
Editus Luxembourg. 2018. Available at: https://www.editus.lu/

Eurostat. 2018a. Eu labour force survey - methodology. Available at: https://ec.europa.eu/eurostat/statistics-explained/index.php/EU_labour_force_survey__methodology (Accessed February 2021).

Eurostat. 2018b. High-tech industry and knowledge-intensive services. Available at: https://ec.europa.eu/eurostat/cache/metadata/en/htec_esms.htm (Accessed February 2021).

Fan, W., and Z. Yan. 2010. "Factors affecting response rates of the web survey: A systematic review." Computers in Human Behavior (2): 132-139. DOI: https://doi.org /10.1016/j.chb.2009.10.015.

Felderer, B., A. Kirchner, and F. Kreuter. 2019. "The Effect of Survey Mode on Data Quality: Disentangling Nonresponse and Measurement Error Bias.” Journal of Official Statistics, 35(1): 93-115. DOI: https://doi.org/10.2478/jos-2019-0005.

Fricker, S., M. Galesic, R. Tourangeau, and T. Yan. 2005. “An experimental comparison of web and telephone surveys." Public Opinion Quarterly, 69(3): 370-392. Available at. https://academic.oup.com/poq/article-abstract/69/3/370/1941667?redirectedFrom=fulltext (accessed February 2021).

Garrouste, C.L., and M. Rodrigues. 2014. "Employability of young graduates in Europe." Journal of Manpower, 35(4): 425-447. DOI: https://doi.org/10.1108/IJM-05-20130106.

Grande, E.D., and A.W. Taylor. 2010. "Sampling and coverage issues of telephone surveys used for collecting health information in Australia: results from a face-to-face survey from 1999 to 2008." BMC Medical Research Methodology, 77(10). DOI: https:// doi.org/10.1186/1471-2288-10-77.

Greene, J., Speizer, H., and W. Wiitala. 2008. "Telephone and web: Mixed-mode challenge." Health Services Research, 43(1 Pl): 230-248. DOI: https://doi.org/10.1111/j.1475-6773. 2007.00747.x.

Groves, R.M. 2005. Survey errors and survey costs, (2nd ed.). Hoboken, New Jersey: John Wiley \& Sons.

Helppie-McFall, B., and J.W. Hsu. 2017. "A test of web and mail mode effects in a financially sensitive survey of older Americans." Journal of Economic and Social Measurement, 42(2): 151-169. DOI: https://doi.org/10.3233/JEM-170444.

Iacus, S.M., G. King, and G. Porro. 2011. "Multivariate Matching Methods That Are Monotonic Imbalance Bounding." Journal of the American Statistical Association, 106(493): 345-361. DOI: https://doi.org/10.1198/jasa.2011.tm09599.

Iacus, S.M., G. King, and G. Porro. 2012. "Causal Inference without Balance Checking: Coarsened Exact Matching.” Political Analysis, 20(1): 1-24. DOI: https: //doi.org/ 10.1093/pan/mpr013.

Iacus, S.M., G. King, and G. Porro. 2018. Package cem. Available at: https://cran.rproject.org/web/packages/cem/cem.pdf (accessed February 2021).

ICLS Resolution, 19th. 2013. Resolution concerning statistics of work, employment and labour underutilization. Available at: https://www.ilo.org/wcmsp5/groups/public/dgreports/—stat/documents/normativeinstrument/wcms_230304.pdf (accessed February 2021). 
Imbens, G.W. 2004. "Nonparametric Estimation of Average Treatment Effects Under Exogeneity: A review." The Review of Economics and Statistics, 86(1): 4-29. DOI: https://doi.org/10.1162/003465304323023651.

Körner, T., and A. Liersch. 2014. Case study on mode effects in the German Labour Force Survey. Deliverable for work package III of the ESSnet on Data Collection for Social Surveys Using Multiple Modes. Available at: http://www.cros-portal.eu/content/ data-collection (accessed December 2014).

Liaw, A., and M. Wiener. 2002. "Classification and Regression by randomForest." $R$ News, 2(3): 18-22. Available at: https://www.researchgate.net/profile/Andy_Liaw/publication/228451484_Classification_and_Regression_by_RandomForest/links/53fb24cc0cf20a45497047ab/Classification-and-Regression-by-RandomForest.pdf (accessed February 2021).

Lipps, O., N. Pekari, and C. Roberts. 2013. Coverage and nonresponse errors in an individual register frame based Swiss telephone election study. FORS. Available at: https://serval.unil.ch/resource/serval:BIB_7865820E3A4F.P001/REF.pdf (accessed February 2021).

Long, J.S., and J. Freese. 2006. Regression models for categorical dependent variables using Stata, (2nd edition). Stata press.

Lugtig, P., G.J.L.M. Lensvelt-Mulders, R. Frerichs, and A. Greven. 2011. "Estimating Nonresponse Bias and Mode Effects in a Mixed-Mode Survey." International Journal of Market Research, 53(5): 1-16. DOI: https://doi.org/10.2501/IJMR-53-5-669-686.

Luiten, A., and K. Blanke. 2015. "Conclusions of the ESSnet - DCSS on web and mixed mode data collection in official social surveys." Conference of New Techniques and Technologies for Statistics (NTTS 9-13 March 2015. Brussels, Belgium. DOI: https://doi.org/10.2901/EUROSTAT.C2015.001.

Manfreda, L.K., M. Bosnjak, J. Berzelak, I. Haas, and V. Vehovar. 2008. "Web Surveys versus other Survey Modes: a Meta-Analysis Comparing Response Rates." International Journal of Market Research, 50(1): 79-104. DOI: https://doi.org/10 $.1177 / 147078530805000107$.

Mathur, M.B., P. Ding, and T.J. VanderWeele. 2018. Package EValue. Available at: https://cran.r-project.org/web/packages/EValue/EValue.pdf (accessed February 2021).

Meinfelder, F. 2014. "Multiple Imputation: An attempt to retell the evolutionary process." AStA Wirtschafts- und Sozialstatistisches Archiv, 8(4): 249-267. DOI: https://doi.org/10.1007/S11943-014-0151-8.

Mercer, A.W., F. Kreuter, S. Keeter, and E.A. Stuart. 2017. "Theory and Practice in Nonprobability Surveys: Parallels between Causal Inference and Survey Inference." Public Opinion Quarterly, 81: 250-279. DOI: https://doi.org/10.1093/poq/nfw060.

Morgan, S.L., and C. Winship. 2007. Counterfactuals and Causal Inference: Methods and Principles for Social Research. Cambridge University Press.

Morris, T.P., I.R. White, and P. Royston. 2014. "Tuning multiple imputation by predictive mean matching and local residual draws.” BMC Medical Research Methodology, 14. DOI: https://doi.org/10.1186/1471-2288-14-75.

Pohjanpää, K. 2014. The report of web pilot study of LFS (WPIII). Statistics Finland. The ESSnet project on Data Collection for Social Surveys using Multiple Modes. 5 September, 2014, Wiesbaden, Germany. Available at: https://ec.europa.eu/eurostat/ 
cros/content/workshop-\%E2\%80\%9Cdata-collection-social-surveys-using-multiplemodes\%E2\%80\%9D-dcss_en (accessed October 2019).

Riillo, C.A.F. 2017. Beyond the question "Does it pay to be green?": How much green? and when?" Journal of Cleaner Production, 141: 626-640. DOI: http://dx.doi.org/ 10.1016/j.jclepro.2016.09.039.

Roberts, C., and C. Vandenplas. 2017. "Estimating Components of Mean Squared Error to Evaluate the Benefits of Mixing Data Collection Modes." Journal of Official Statistics, 33(2): 303-334. DOI: https://doi.org/10.1515/jos-2017-0016.

Rosenbaum, P.R. 2005. "Sensitivity Analysis in Observational Studies.” In Encyclopedia of Statistics in Behavioral Science, edited by B.S. Everitt and D.C. Howell, 1451-1462. John Wiley \& Sons, Ltd, Chichester.

Rosenbaum, P.R., and D.B. Rubin. 1983. "The central role of the propensity score in observational studies for causal effects." Biometrika, 70(1): 41-55. DOI: https://doi.org/10.1093/biomet/70.1.41.

Sarracino, F., C.A.F. Riillo, and M. Mikucka. 2017. "Comparability of web and telephone surveys for the measurement of subjective well-being." Survey Research Methods, 11(2): 141-169. DOI: https://doi.org/10.18148/srm/2017.vlli2.6740.

Schonlau, M., K. Zapert, L.P. Simon, K. Sanstad, S. Marcus, J. Adams, M. Spranca, H. Kan, R. Turner, and S.H. Berry. 2003. "A Comparison Between Responses From a Propensity-Weighted Web Survey and an Identical RDD Survey." Social Science Computer Review, 21(10): 1-11. DOI: https://doi.org/10.1177/0894439303256551.

Schouten, B., and J. van der Laan. 2014. ESSnet deliverable WPIII: Mode effect decompositions for the Dutch Labour Force Survey. Deliverable for work package III of the ESSnet on Data Collection for Social Surveys Using Multiple Modes. 5 September, 2014, Wiesbaden, Germany. Available at: https://ec.europa.eu/eurostat/cros/content/workshop-\%E2\%80\%9Cdata-collection-social-surveys-using-multipleQ15modes\%E2\%80\%9D-dcss_en (accessed October 2019).

Sentas, P., and L. Angelis. 2006. "Categorical missing data imputation for software cost estimation by multinomial logistic regression.” Journal of Systems and Software, 79(3): 404-414. DOI: https://doi.org/10.1016/j.jss.2005.02.026.

Shrier, I., R.W. Platt, and R.J. Steele. 2007. Re: "Variable Selection for Propensity Score Models.” American Journal of Epidemiology, 166(2): 238-239. DOI: https://doi.org/10.1093/aje/kwj149.

Sidney, J.A., C. Coberley, J.E. Pope, and A. Wells. 2015. "Extending coarsened exact matching to multiple cohorts: an application to longitudinal well-being program evaluation within an employer population." Health Services and Outcomes Research Methodology, 15(2): 136-156. DOI: https://doi.org/10.1007/S10742-014-0136-7.

Sinclair, M., J. O'Toole, M. Malawaraarachchi, and K. Leder. 2012. "Comparison of response rates and cost-effectiveness for a community-based survey: postal, internet and telephone modes with generic or personalised recruitment approaches." $B M C$ Medical Research Methodology, 12(1): 132. DOI: https://doi.org/10.1186/1471-228812-132.

Sousa-Poza, A., and A.A. Sousa-Poza. 2000. "Well-being at work: a cross-national analysis of the levels and determinants of job satisfaction." The Journal of SocioEconomics, 29(6): 517-538. DOI: https://doi.org/10.1016/S1053-5357(00)00085-8. 
Stuart, E.A. 2010. "Matching Methods for Causal Inference: A Review and a Look Forward." Statistical Science, 25(1): 1-21. DOI: http://dx.doi.org/10.1214/09-STS313. Ting, Y. 1997. "Determinants of Job Satisfaction of Federal Government Employees." Public Personnel Management, 26(3): 313-334. DOI: https://doi.org/10.1177/ 009102 609702600302.

Tourangeau, R., L.J. Rips, and K. Rasinski. 2000. The psychology of survey response. Cambridge, UK: Cambridge University Press.

Van Buuren, S. 2012. Flexible imputation of missing data. New York, NY: Taylor \& Francis Ltd.

Van Buuren, S., and K. Groothuis-Oudshoorn. 2011. "mice: Multivariate Imputation by Chained Equations in R.” Journal of Statistical Software, 45(3). DOI: http://dx.doi.org/ 10.18637/jss.v045.i03.

VanderWeele, T.J., and P. Ding. 2017. "Sensitivity Analysis in Observational Research: Introducing the E-value.” Annals of Internal Medicine, 167(4): 268-274. DOI: https:// doi.org/10.7326/M16-2607.

Vannieuwenhuyze, J.T.A., G. Loosveldt, and G. Molenberghs. 2014. "Evaluating Mode Effects in Mixed-Mode Survey Data Using Covariate Adjustment Models." Journal of Official Statistics, 30(1): 1-21. DOI: https://doi.org/10.2478/jos-2014-0001.

Vila, L.E., and B. Garcia-Mora. 2005. "Education and the Determinants of Job Satisfaction." Education Economics, 13(4): 409-425. DOI: https://doi.org/10.1080/ 09645290500251730.

Ye, C., Fulton, J., and R. Tourangeau. 2011. "More positive or More Extreme? A MetaAnalysis of Mode Differences in Response Choice." Public Opinion Quarterly, 75(2). DOI: https://doi.org/10.1093/poq/nfr009.

Received April 2019

Revised October 2019

Accepted September 2020 